- EDTTORIAL • PULSANDO LA CUESTIÓN SOBRE LA VIVIENDA Y EL HÁBITAT / PRESSING THE HOUSING AND HABTTAT QUESTION, Rosa María Añón Abajas • ENTRE LínEAS • THE PRIMACY OF RESIDENTIAL QUALITY IN URBAN CREATION. A CURRENT OBSERVATION ON A RECURRING NOTION / LA PRIMACÍA DE LA CALIDAD RESIDENCIAL EN LA CREACIÓN URBANA UNA OBSERVACIÓN ACTUAL DE UNA NOCIÓN RECURRENTE. Sten Gromarko • ARTÍCULOS • HACIA LA NUEVA SOCIEDAD COMUNISTA: LA CASA DE TRANSICIÓN DEL NARKOMFIN, EPILOGO DE UNA INVESTIGACIÓN / TOWARDS THE NEW COMMUNIST SOCIETY: THE TRANSITION HOUSE OF NARKOMFIN, A RESEARCH EPILOGUE. Daniel Movilla Vega; Carmen Espegel AlonSO • EL SISTEMA COMO LUGAR. TRES ESTRATEGIAS DE COLECTIVIZACIÓN DEL ESPACIO DOMÉSTICO CONTEMPORÁNEO I THE SYSTEM AS A PLACE. THREE COLLECTIVIZATION STRATEGIES OF THE CONTEMPORARY DOMESTIC SPACE. Fernando Nieto Fernández • LA CASA EN "CAMPO DE ARROZ". UN IDEOGRAMA DE INTERACCIÓN EN EL HÁBITAT JAPONÉS CONTEMPORÁNEO / HOUSE IN A "RICE PADDY". AN IDEOGRAM OF INTERACTION IN THE CONTEMPORARY JAPANESE HABITAT. Pedro Luis Gallego Fernández • LA CONSTRUCCIÓN TEORICA Y PRÁCTICA DE UN NUEVO HÁBTTAT MODERNO: UNOS PATIOS Y UNA CALLE (1946-1954) THE THEORETICAL AND PRACTICAL CONSTRUCTION OF A NEW MODERN HABITAT: SOME PATIOS AND A STREET (1946-1954). Juan Pedro Sanz Alarcón, Miguel Centellas Soler, Pedro García Martínez • EL BARRIO DE SÃo VICTOR DE ÁLVARO SIZA: ENTRE LA TEORÍA Y LA PRÁCTICA DE LAS OPERACIONES SAAL / DISTRICT OF SÃO VICTOR OF ÁLVARO SIZA: BETWEEN THEORY AND PRACTIC OF THE SAAL OPERATIONS. Aitor Varea OrO • REVISITING PUBLIC SPACE IN POST-WAR SOCIAL HOUSING IN GREAT BRITAIN REPENSANDO EL ESPACIO PÚBLICO DE LAS VIVIENDAS SOCIALES DE POST-GUERRA EN GRAN BRETAÑA. Pablo Sendra • INTIMIDADES TRANSGREDIDAS: HABITAR EN TRÁNSITO / TRANSGRESSED PRIVACIES: TO LIVE IN TRANSIT. Maŕa Prieto Peinado • RESEÑAS BIBLIOGRÁFICAS • R.D. MARTIENSSEN: LA IDEA DEL ESPACIO EN LA ARQUITECTURA GRIEGA. Juan Luis Trillo de Leyva

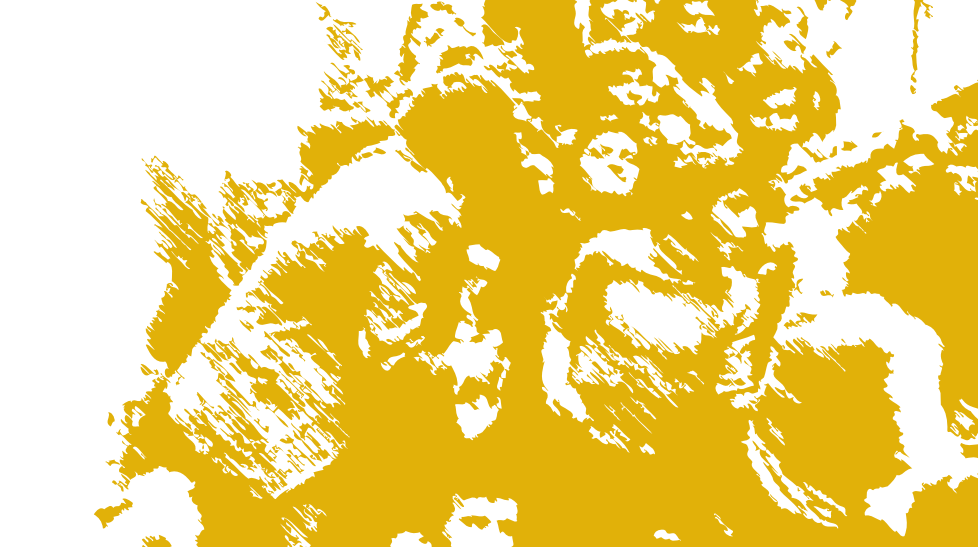

(n)
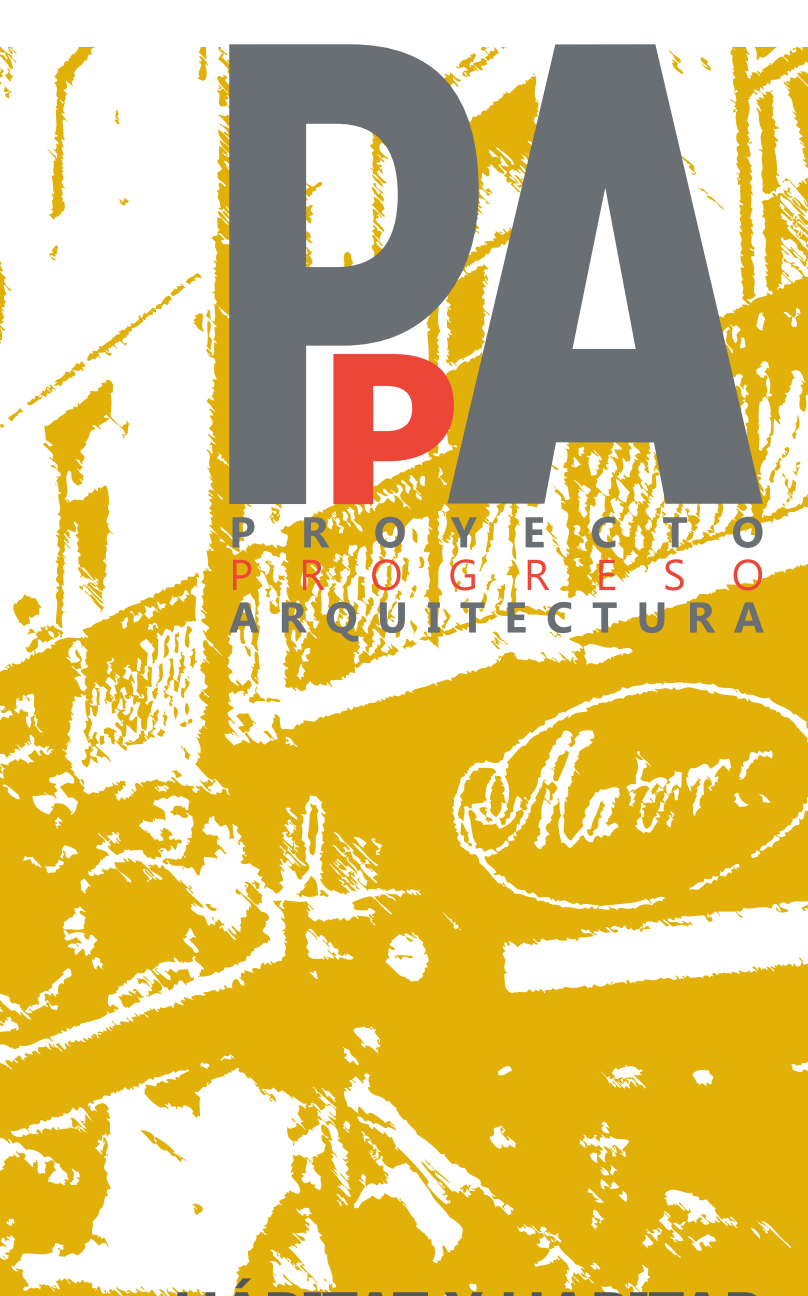

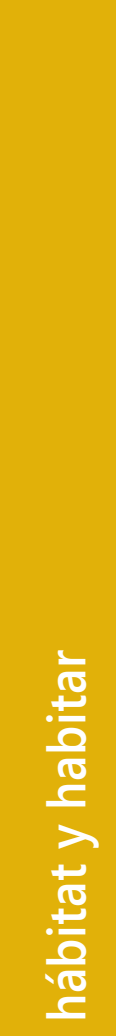

N9 


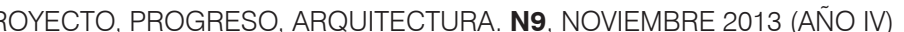

hábitat y habitar

editorial
PULSANDO LA CUESTIÓN SOBRE LA VIVIENDA Y EL HÁBITAT / PRESSING THE HOUSING AND HABBTAT QUEESTION
Rosa María Añón Abajas

entre líneas

THE PRIMACY OF RESIDENTIAL QUALITY IN URBAN CREATION. A CURRENT OBSERVATION ON A RECU UNAen GrOBSERVACION ACTUAL DE UNA NOCIÓN RECURRENTE

artículos

HACIA LA NUEVA SOCIEDAD COMUNISTA: LA CASA DE TRANSICIÓN DEL NARKOMFIN, HOUSE OF NARKOMFIN A RESEARCH EPILOGUE

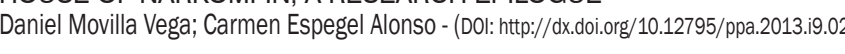

EL SISTEMA COMO LUGAR. TRES ESTRATEGIAS DE COLECTIVIZACIÓN DEL ESPACIO

STRATEGIES OF THE CONTEMPORARY DOMESTIC SPACE

LA CASA EN “CAMPO DE ARROZ”. UN IDEOGRAMA DE INTERACCIÓN EN EL HÁBITAT

LA CASA EN "CAMPO DE ARROZ". UN IDEOGRAMA DE INTERACCIÓN EN EL HÁBITAT
JAPONES CONTEMPORÁNEO / HOUSE IN A "RICE PADDY". AN IDEOGRAM OF INTERACTION IN THE CONTEMPORARY JAPANESE HABITAT

LA CONSTRUCCIÓN TEORICA Y PRÁCTICA DE UN NUEVO HÁBITAT MODERNO: UNOS PATIOS Y UNA CALLE (1946-1954) / THE THEORETICAL AND PRACTICAL CONSTRUCTION OF A NEW MODERN

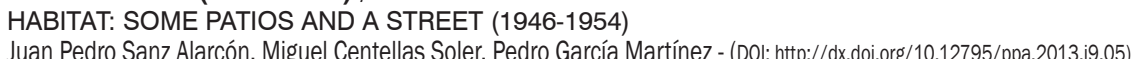

EL BARRIO DE SÃO VICTOR DE ÁLVARO SIZA: ENTRE LA TEORÍA Y LA PRÁCTICA DE LAS OPERACIONES SAAL / SÃO VICTOR DE ÁLVARO SIZA DISTRICT: BETWEEN THEORY AND PRACTICE OF

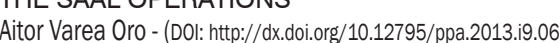

REVISITING PUBLIC SPACE IN POST-WAR SOCIAL HOUSING IN GREAT BRITAIN/ REPENSANDO EL ESPACIO PUBLICO DE LAS VIVIENDAS SOCIALES DE POST-GUERRA EN GRAN BRETAÑA

INTIMIDADES TRANSGREDIDAS: HABITAR EN TRÁNSITO / TRANSGRESSED PRIVACIES: TO LIVE IN

TRANSIT

eseña bibliográfica TEXTOS VIVOS

R.D. MARTIENSSEN LA IDEA DEL ESPACIO EN LA AROUITECTURA GRIEGA

Juan Luis Trillo de Leyva 


\section{LA CONSTRUCCIÓN TEÓRICA Y PRÁCTICA DE UN NUEVO \\ HÁBITAT MODERNO: UNOS PATIOS Y UNA CALLE (1946-1954)}

THE THEORETICALAND PRACTICAL CONSTRUCTION OF A NEW MODERN HABITAT: SOME

PATIOS AND A STREET (1946-1954)

Juan Pedro Sanz Alarcón; Miguel Centellas Soler; Pedro García Martínez
EL GERMEN DE LA CIUDAD MODERNA: DE LA

"MÁQUINA DE VIVIR" A LA CARTA DE ATENAS

E I contenido teórico y práctico que la arquitec-

tura moderna venia desarrollando a lo largo de los primeros años del siglo XX y sobre todo con una clara mayor intensidad durante los anos veinte, tendria una fecha señalada en 1928. La clia, en junio de ese año, en el castillo de La Sarraz en Suiza, organizada por Le Corbusier principalmente, junto con Sigfried Giedion daria forma a lo que se conoció como CIAM (Congrés internationaux d Architecture Moderne), en su primera edción, a la que concurmeron casiuna treintena de arquitectos. Los debates desarrollados posteriormente a través de los sucesivos CIAM senillan de importante referenci para el avance de la disciplina arquitectónica.

Los manifestos isori mos junto con los ejemplos prácticos desarrollaron un manual arquitectónico que incidio mente, como la globalización de la arquitectura La casa era una "máquina de vivir" que en un primer momento secos de funcionamiento en el sentido más amplio de la palabra. Bajo estas premisas tendira lugar en Frankfurt en 1929 el CIAM II, denominado como The Existenzminimum en el que se regularon la metrica, la organización interiory el programa de minimos para los proyectos de vivendas destinadas a la clase obrera, los cuales iban a ser los encargados de conformar la ciudad contemporanea. Tras este evidente avance en los modelos, las teorías modernas sobre el espacio domestico se ven reconfiguradas de forma clara tras el CIAMIV celebrado a bordo del Patris por aguas del Mediterráneo en el verano de 1933.

El resultado final de dicho congreso fue la elaboración de la conocida "Carta de Atenas". Uno de sus conten dos, de induabable trascendencia en los planteamientos arquitectónicos, es la "mediterraneización de la arquitecde los parámetros particulares de cada lugar: el clima, la

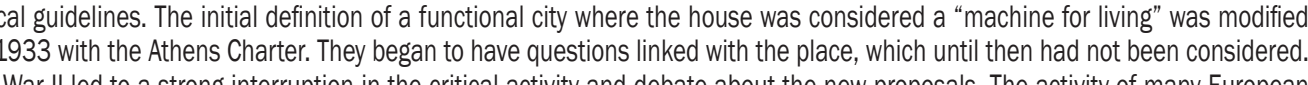
nexample in the United States, favoured new lines of work. His 1946 proposals in the Peruvian city of Chimbote showed "a more human" concern than the previous examples. The grant leap in these approaches took place in the early 50 s when the young collaborators of Le Corbusier were entrusted with the

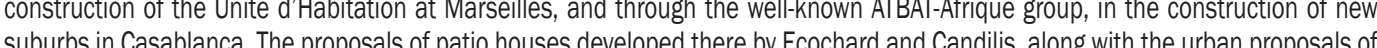
the Smithsons in London, came together in the CIAM IX that brought about the creation of TEAM 10. This was the turning point and the start towards a new concept of the contemporary habitat.

Persona de contacto / Corresponding author. juan.sanz@upct.es. Escuela de Arquitectura e Ingeniería de Edificación. Universidad Politécnica de Cartagena

1. La presencia de Serty Le Corbusier como figuras principales del CIAM IV daría lugarar a la publicación posterior por parte de ellos de los textos resumen de contenido del congresos. De esta forma Sert en 1942 publicó bajo el tituluo Can Our Cities Survive? su particular versión de lo acontecido y Le Corbusier un aîn más tarde, en 1943, publicó su propia versión de los extensos debates del CIAM IV con el tiftulo de La Charte d'Athenes. Dichos textos se conviritieron en los

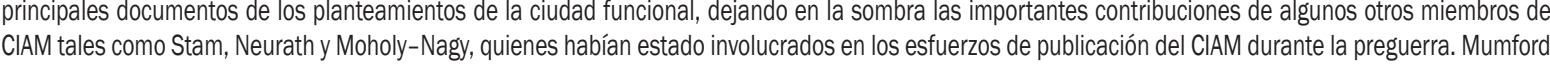
(19)

2 Morales. José: La disolución de la estancia. Transformaciones domésticas 1930-1960. Madrid: Rueda, 2005. 


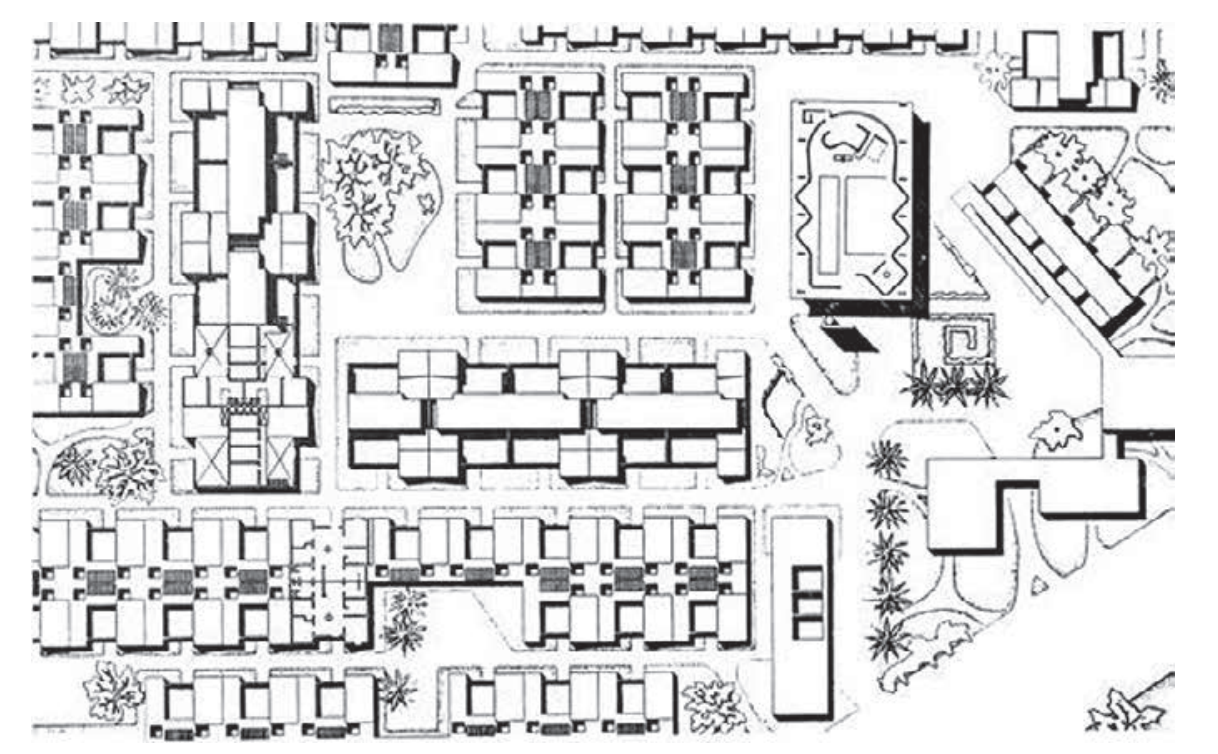

opografía o la orientación. Se trata, por lo tanto, del inicio de una preocupación por lo local, lo cercano y lo vernáculo. Un germen de la "desinternacionalización" de la arquitectura en cierto modo. Pero la definición de la ciudad influida por las conclusiones de la exposición de Cornelis van Esteren ${ }^{3}$ " "La idea de la ciudad funcional" celebrada años antes, en 1928, otorgó la característica más clara y contundente a los nuevos planteamientos. La ciudad quedaba delimitada bajo los parámetros de la zonificación, como islas funcionales, perdiéndose definitivamente el carácter intrínseco de la ciudad tradicional donde confluian con libertad, armonia y riqueza las interrelaciones de toda indole. Aparecia por lo tanto el "guión" arquilectónico definitivo que dejaba de lado la ciudad histórica apostando por la separación de usos y que fue el modelo a seguir hasta que comenzó la Segunda Guerra Mundia en el año 1939

EL PATIO DE LA COMUNIDAD: SERT Y LAS BASES DE UN NUEVO HÁBITAT

La Guerra ocasionó el exilio, sobre todo a Estados Unidos, de muchos de los miembros de mayor relevancia de los CIAM. En ese contexto, Gropius, Sert y Giedion, realizaron un gran esfuerzo para establecer de nuevo los encuentros, reuniones y debates. La posición docente de varios de ellos propició la divulgación de las conclusiones obtenidas con anterioridad con una fuerte repercusión Cabe recordar que el estuerzo más destacado lue la publicación en 1942 de Sertdel libro Can Our Cities Sunvive?

estructurado bajo la diferenciación de las cuatro famosas funciones de la ciudad establecidas en aquel encuentro de 19334. La ciudad dividida en vivienda, trabajo, ocio y circulación

Al finalizar la Segunda Guerra Mundial, en 1945, estos parametros que habian definido la ciudad anteriormente iban a quedar un tanto descontextualizados. Los planes de posguerra para la reconstrucción de las ciudades y as nuevas viviendas como grandes alojamientos de las masas obreras, debian tener un nuevo caracter ${ }^{5}$. Tras la discontinuidad y la irregularidad provocada por el exilio en tomo a las reuniones de los CIAM, los encuentros se retomarian despues del fin del conticto belico. De este modo, los ClAM Vly Vil, celebrados en 1947 en Bridgwaler (Inglaterra) y en Bergamo (Italia) en 1949, respectivamente, sirvieron para trabajar sobre las bases de nuevos planteamientos arquitectónicos.

Sert asumió en el CIAM VI la dirección de la organizaciôn y ocupó el puesto dejado por el holandés Cornelis van Eesteren. La figura de Sert, curiosamente, iba a representar una segunda versión de la arquitectura como propuesta en relación con los sistemas (culturales, climáticos, artesanales...) locales, anticipada e incluida en e CIAM IV de 1933 por el mismo, en lo que anteriormente hemos cilado como un vinculo de la arquitectura con el medio mediterráneo. Pruebas manifiestas de la postura de Sert y que sirven de ejemplo instrumental para perin su posicion en el debare arquilectunico, son los planes de desarrollos urbanos para Lima y sobre todo

3. Somer, Kees: The Functional city: the CIAM and Cornelis van Eesteren, 1928-1960. The Hague: EFL Foundation, 2007 4. Sert, José Luis: Can our cities survive? : An ABC of urban problem, their analysis, their solutions; based on the proposals formulated by the CIAM. Cambridge:
Harrard University Press, 1944 . el de Chimbote, en Perú (1946-1948) realizados junto Paul Lester Wiener (figura 1). En éste último, basado en la creación de una nueva ciudad portuaria en relación con los recursos mineros del none de Perú, a partir de un pequéro pueblo de pescadores existente a borde de a pesar de consenarse la zonificacín funciona arida

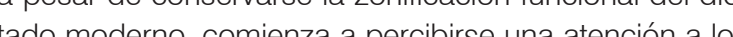
medios cercanos En él se explo un construcción autóctona y la inclusión de patios, a diferente escalay usocomo revisín contempoŕn y moderna de la aruitecura verńc espuesta La componente social de la arquitectura entra en juego. Para ello, Setc cré a tavís de patios en la escala urbana uns plazas unos centso civicos en los a se propiciaba el nazentroy lacrecín de la comunidad. Los patios senví́n de núcleo urbano, donde la esLos a manas y el caŕcter más sociológico que en los nuevos planteamientos arquitectónicos comenzaban a emerger Ese centro cívico era una gran plaza central delimitada perimetralmente por edificaciones en contraposición con a idea de bloque abierto que hasta el momento se ha bía desarrollado en la mayoría de ejemplos modernos Los equipamientos públicos (la iglesia, la biblioteca el museo) definían el espacio urbano que conectaba su vez con las zonas comerciales que incluían de nuevo pequeños patios. La transición espacial a través de un juego de zonas cubiertas y descubiertas, patios cerrados y semiabiertos, plazas y viales públicos, enriquecía sin duda alguna el planteamiento comunitario del espacio público. Fue la primera muestra de colectividad interpretada, no tanto desde la funcionalidad ortodoxa sino desde el conocimiento de los parámetros configuradores de una sociedad en un lugar y momento concreto perfectamente determinado. En la escala doméstica, el realojo de los habitantes de las chabolas del poblado

xistente, se realizaba a través de casas patio que suponían una nueva relectura, no solo de la casa mediterránea como referente, sino de la forma de habitar tradcional en la zona de actuación 6 . El sistema constructivo a través de muros de estas viviendas las hacía viables de acuerdo con la escasez de medios locales. La vivienda se organizaba en forma de "U" entorno a un gran patio que sevra de núcleo de aniculach y organización de ban y tenín accoso desde á Los muros perimetahacia la calle se mostraban précticamente opacos sin apenas huecos a la ví pública entelizando el corácter primordial de patio La agrupación de viviendas permitra un desarollo extensivo de a nuevaciudad en vinculaćn directa con el medio rurat aprovechando el patio para habitual vida en el exterior propia de estas zonas climá ticas Este planteamiento de "tapiz urbano" constituyó primer ejemplo de hábitat de alta densidad y baja altura que daba una respuesta contundente al problema de infravivienda y los nuevos desarrollos de realoio on ciudades donde eran implanteables las idilicas y utópicas mácenes del espacio público natural de la ciudad funcional de bloques aislados Se trató por lo tanto de un inicial interpretación de los medios autóctonos bajo una nueva óptica matizada de la ciudad moderna. Todo ello quedó reflejado como texto definitorio en el artículo de Sert publicado tan solo unos años después, en agosto de 1953, en la revista Architectural Forum bajo el título "Can Patios Make Cities?" El El Plan de Chimbote fue pre sentado en el CIAM VII de 1949 celebrado en Bérgamo al que asistieron por primera vez una nueva generación de arquitectos que iban a ser los encargados de pautar a partir de ese momento el futuro de la arquitectura. Posteriormente también, con motivo del CIAM VIII, celebrado en Hoddesdon (Inglaterra) en 1951, Sert, a través del propio lema del congreso "Heart of the City" manifestó claramente sus planteamientos e intereses.

5. Nada más acabar l l Guerra en Europa, Giedion escribióa Le Corbusier desde Harvard con su propuesta para eI CIAM VI: "Let me know if you also agree tha

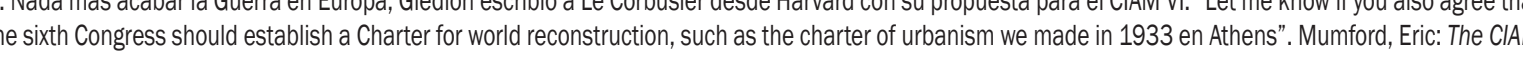
discourse on urbanism, 1928-1960. Cambridge: MIT Press, 2000.

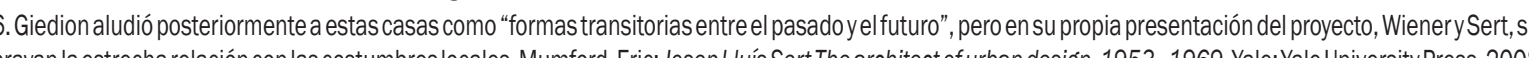
7. Sert. José Luis: “Can patios make cities?? En Architectural Forum. No 99, 1953. P. .124-131. 


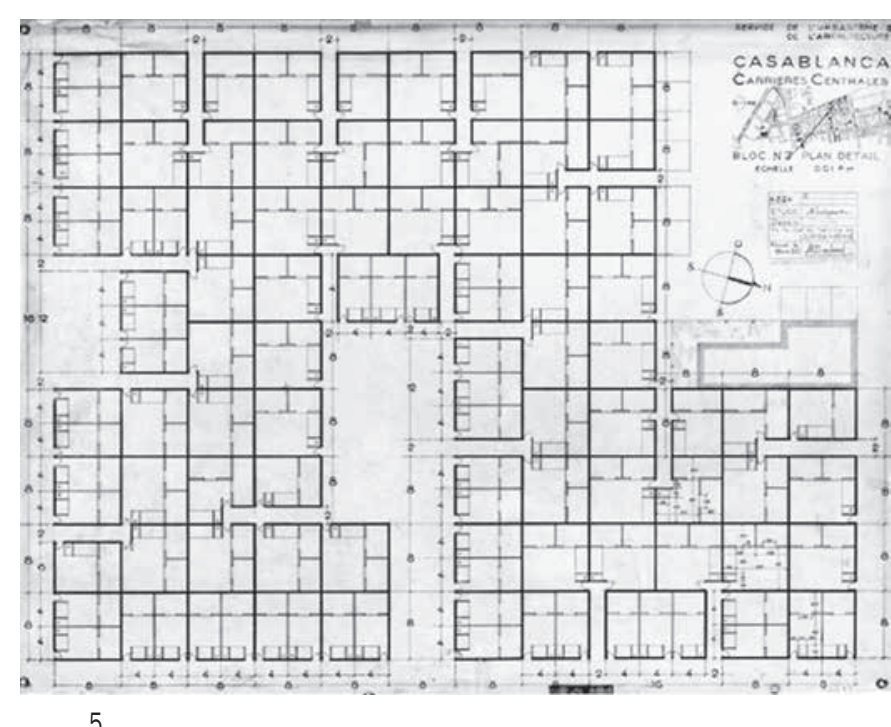

5

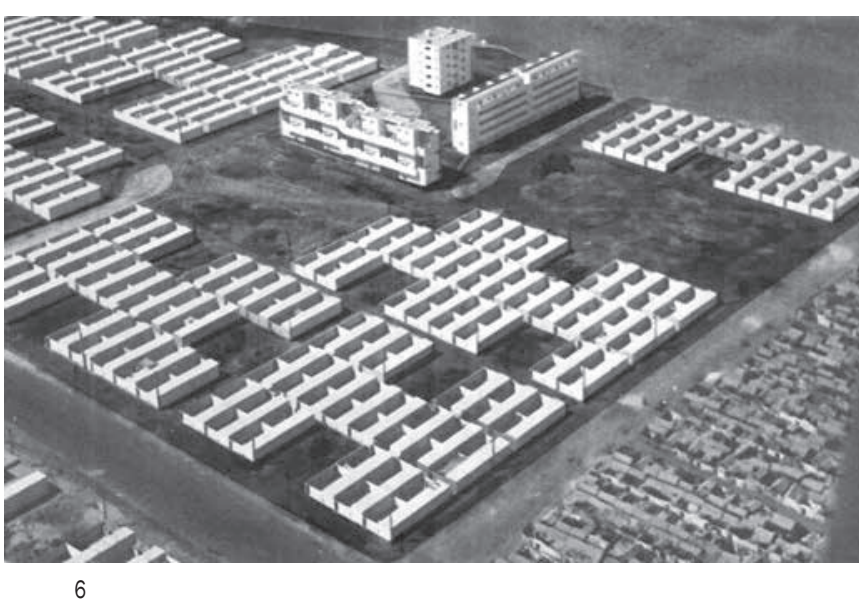

La célula mínima se disponía como un conjunto de cuatro viviendas creando un sistema de agrupación de mayor tamaño con una escala mucho más urbana (figuras 5 y 6 ). Este grupo permitía tener una calle de acceso a las cuatro viviendas que lo definían, que eran a su vez diferentes en tamaño, programa y organización espacial, conservando siempre el invariante de un patio individual. Las diferentes estancias vertían al patio que a su vez servía de separación entre la parte de vivienda y la pequeña zona de servicio. Los muros exteriores servían de elementos de yuxtaposición de las diferentes viviendas que eran opacos mientras que los cerramientos del patio contenían los huecos de iluminación y ventilación La vivienda tipo se organizaba bajo un modulo de ocho

9. "El patio no es tan sólo un elemento totalmente principal en la historia de la arcuitectura (...); es también la base de un verdadero sistema de composición,

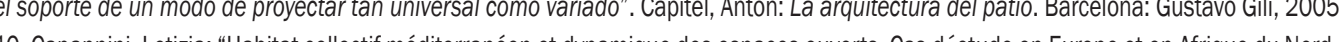

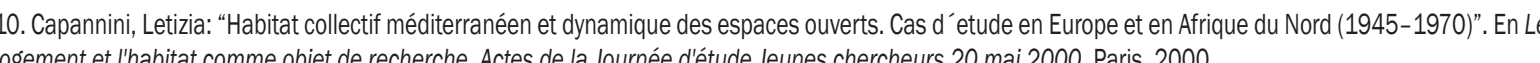
chard en los Carrieres Centrales de Eosoblanca. 7. Cas

por ocho metros incluyendo al patio (figura 7). Normal(le planteaba en forma de "L" sobre espacio privado exterior. La combinación de dichos paquetes de viviendas, en la escala jerárquica superio ciones de contorno en lo que a la implantación se refiere. De esta forma el patio como concepto de interpretacón del espacio arquitectónico y a través de sus diferenles utilizaciones (escalas, usos, geometrís, ) propicia una relectura continua del pasado generando una herra(1)

Este mecanismo de ciudad horizontal habí sido de sarrollado con anterioridad en las conocidas propuestas de Hilberseimer mediante agrupación de viviendas en "L" a finales de los años veinte y principio de los treinta, en el menos estudiado planteamiento de Diotallevi, Marescotti y Pagano de su Cittá Orizzontale en la periferia de Milán en 1938, que desarrollaba un catálogo métrico y programático de las diferentes posibilidades de viviendas también en "L" entorno a un patio Cabe destacar que ambos proyectos partían de un módulo de vivienda tipo sin existir un sistema de agrupaciones de mayor escaa. El planteamiento era de una ciudad horizontal unitaria casi abstracta con menor capacidad de adaptación a las condiciones del entorno que la propuesta de Casablanca. Posteriormente este desarrollo de Ecochard para los Carriéres Centrales serviría de pauta de partida para el conocido proyecto de Adalberto Libera para la ciudad hoirontal de Tuscolano en Roma, que desarrolló en 1954 a a vuelta de un viaje a Marruecos donde había conocido estas nuevas propuesta de hábitat colectivo ${ }^{10}$. El grupo de arquitectos del ATBAT-Afrique con Candilis a la cabeza completó la ciudad horizontal de Ecochard con tres bloques de vivienda colectiva en altura como ejemplo

5. Plano de detalle de una agrupación tipo 6. Maqueta de los Carrieres Centrales de

7. para los Carrieres Centrales de Cocchald

didos del ededificio de Candililis y Woods Carrieres Centrales

trame $8 \times 8$

type $8 A^{1}$

impe...e sud
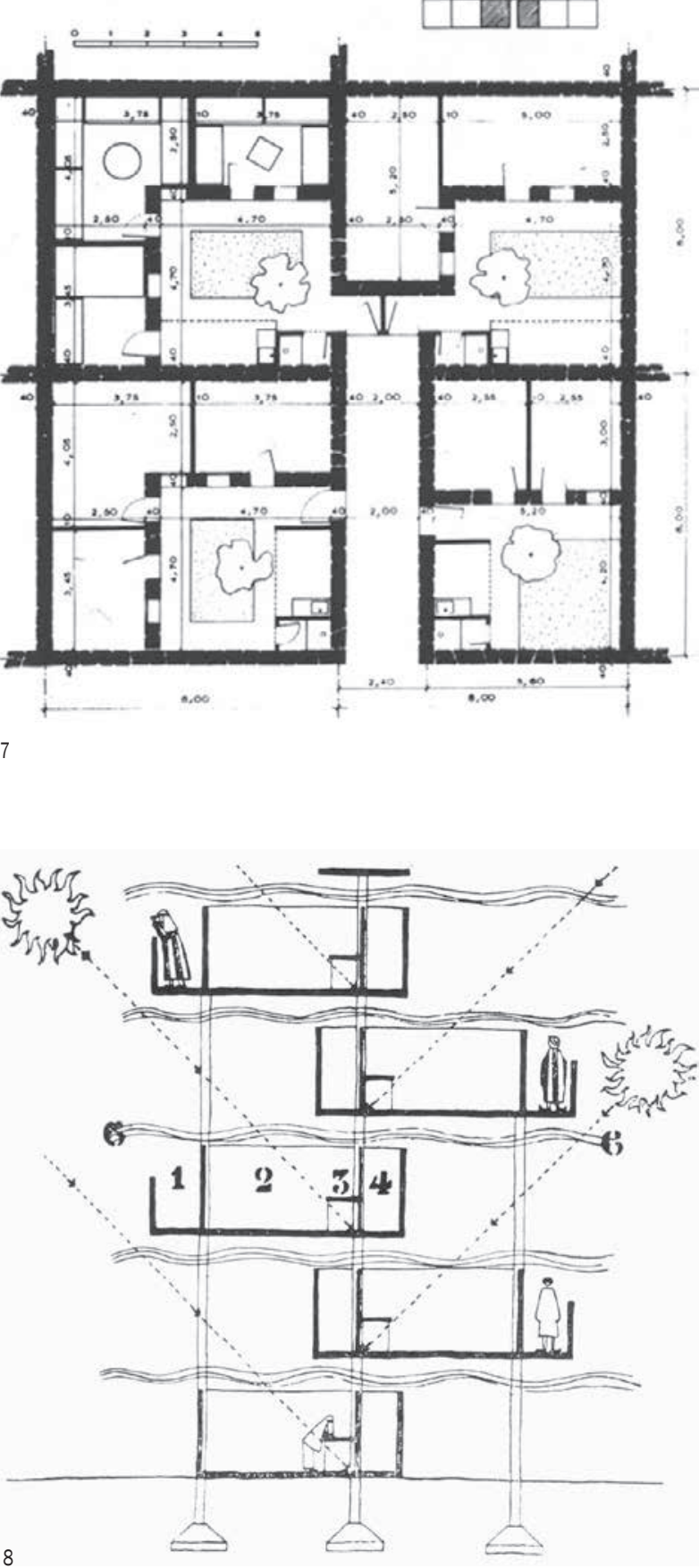

adical de una nueva interpretación de las condiciones loales bajo las pautas que en Europa estaban definiendo as ciudades. Tras la experiencia de Candilis en Marsella se realizó una apuesta por el desarrollo en vertical de la agrupación de viviendas. Se crearon por lo tanto patios Candilis, suspendidos, en lugar del tradicional palio. "en armoní con el medio natural, social, económico y ético"11 (figura 8).

Parece claro que este trabajo determinó un cambio evidente en la práctica de la nueva arquitectura. A partir del año 1953, cuando las propuestas de Ecochard y las del ATBAT-Afrique estaban terminadas iban a concatenarse una serie de acontecimientos que influirían en nuevo rumbo de la disciplina.

NUEVO MARCO TEÓRICO DEL HÁBITAT: EL CIAM IX EN AIX-EN-PROVENCE (1953) Y EL MANIFIESTO DE DOORN (1954)

La repercusión de los experimentos arquitectónicos que se habían desarrollado en Marruecos a principios de los ancer manifiesta. En 1952, a anterior habían decidorticipantes en el CIAM VIII del año en Suecia para preparar el siguiente congreso. Fue notable la asistencia de un gran número de jóvenes arquitectos miembros de los CIAM que posteriormente se agruparían en lo que hemos conocido como TEAM 10 e iban a tomar el relevo de los grandes maestros, entre ellos Jaap Bakema, Aldo van Eyck y Georges Candilis. Resultô destacable la ausencia a su vez de miembros históricos de los CIAM como Le Corbusier, Josep Lluís Sert, Walter Gropius, y Sigfried Giedion. Se encontraron algunos de quienes habían estado criticando las posturas de los CIAM junto con sus métodos desde la Segunda Guerra Mundial y se propició el acuerdo con otros arquitectos que pensaban de modo similar. En este encuentro se puso de manifiesto de forma evidente la ruptura generacional entre las posturas arquitectónicas de los diferentes miembros. El grupo de arquitectos marroquís GAMMA con Candilis a la cabeza, tuvo una especial importancia en los debates. De esta forma se daría paso al CIAM IX

11. Candilis, Georges: "Habitat de type musulman à Casablanca". En L'Architecture d'Aujourd'hui, No 60. Junio 1955. P. 39-41. 
1212 (Assemblée de constructeurs pour une rénovation architecturale), Ios representantes de las colonias francesas ya citados como GAMMA (Groupe d'Architectes Modernes Marocains), el grupo del CIAM Alger o el italiano. El Congreso representó un punto de inflexión

Dos de las rejillas (Grid) que el protocolo establecía

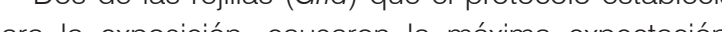
"The Habion "le Mahieddine Grid", ambas diseñadas por los arquitec- n Casablanca que hemos descrito (figuras 9 y 10 ).

Una tercera rillla que atrajo le atención fue Ia "Urban Re identification Grid"12 realizada por Alison y Peter Smithson que analiź́ la vida diaria de la clase obrera del barrio Bethnal Verde de Londres. Esta comprensión del entorno construido por la noción de sus datos sociales represen-

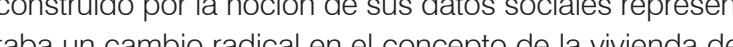
movimiento moderno Las reillas inclúan no solo métodos y datos cuantitativos sino también cualitativos. Era primer manifiesto teórico oficial sobre el nuevo concepto de "hábitat" que sustituía al de "habitación" Para el grupo de Bodiansky y Candilis junto con el resto de integrantes del GAMMA, la idea de alojamiento era entendida como un proceso evolutivo, creciente y adaptable que se veía conformado entorno a la climatología local y a la tecnología al alcance. Las viviendas partían de una infraestructura básica y se proponían como posible autoconstrucción aunque fuera de un modo parcial.

Se trataba de un acercamiento mucho "más humano" de la arquitectura moderna. De nuevo siguio sin concretarse una verdadera "Carta del Hábitat" pero se ejemplificó a través de las exposiciones que hemos señalado como debía ser el camino a seguir en las propuestas de vivienda. Los viejos y nuevos modos de pensar la ciudad quedaban enfrentados. La afinidad mostrada por muchos de los participantes en el debate de Aix-en-Provence propició el encuentro en común, tanto personal como profesional, de varios significados arquitectos. Las soluciones comunes planteadas mostraban una amplia gama de articulaciones formales y marcos teóricos. Y sobre todo reconocían un deseo común por

de Francia en el verano de 1953. Fue el de todos los congresos. Allí asistieron represent varios grupos nacionales y locales de los CIAM. Entre los que podemos destacar: los británicos MARS (Modern 'de 8' y 'Opbouw' el grupo de Le Corbusier, ASCORAL niños jugarían.... Era un nuevo avance mucho más socia crear entornos arquitectónicos que fomentasen y animasen las relaciones entre habitantes, en un edificio y su entorno, siempre en vinculo directo con los condicionantes culurales propios. Alison y Peter Smithson que forporpria dez grapo británico MARS y que asistieron por pís y advez a un Congreso CIAM, mostraron especial Para nura to fue mayor logro y la representación de una

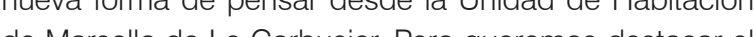

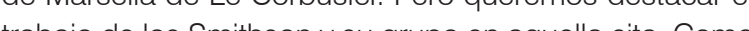
ceñán se anatizan lo e un bario aspectos cotidanos de un lía n lavida te en propuesta la tectónica los arquitectos presentaronenelCongresode Aix-on-Provence el proyecto del Golden tane en Londres (fig 1 11). Todo ese ańlis pautado de las condiciones de vida quiso ejemplicarse. Así se concibió mediante una calle suspendida en elare que articulaba todo el coniunto Esa calle conectaba con el tejido sociocultural del resto de la la Cara de Atenas hár definid. El proyecto se entelazaba con la vida del bario. El corledor se defina con amplitud para aue en el ocurieran actividades popirs del espacio púlico. Era la base idónea para dor lugary cabida as relciones humanas entre los diferentes ha-

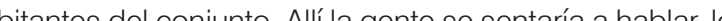
nueva calle queda en fachada, en el exterior, en vínculo directo con el medio circundante. El bloque aislado res mal so habla convertido en un eslabón más de la traiunto la ciudad. Los tamosos fotomontajes del proyecto

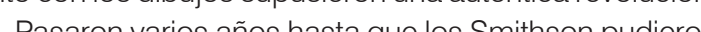
levar a ca estas ios hasta que los Smilithon pudieron rrió a la so estas ideas en un edirio construido. Ocuedificio del Robin Hood Gardens.

Las propuestas de Casablan ay Londes consulueron un soplo de renovacín en el concepro cel hab Quedaban demostradas las tn el concepto del habbitat. bían sido discutidas.

Tras el éxito del CIAM IX, la organización del décimo encuentro celebrado en 1956 iba correr a cargo de los Jonos arquilectos señalados. En los años previos, serie de postulados inicies que hab́n quedado algo inconexos. Estas contribuciones propusieron sustitur erarqú funciona de vivenda trabajo, ocio y transporte que hab́e definido la Carta de Atenas por una clasificación escalar de la comunidad a través de cuatro apartados: casa agrícola aislada, pueblo, ciudades pequeñas de varios tipos (industriales, administrativas, especiales) y grandes ciudades multifuncionales, Su relación con medio, en su hábitat, paso a llamarse la "sección del valle 


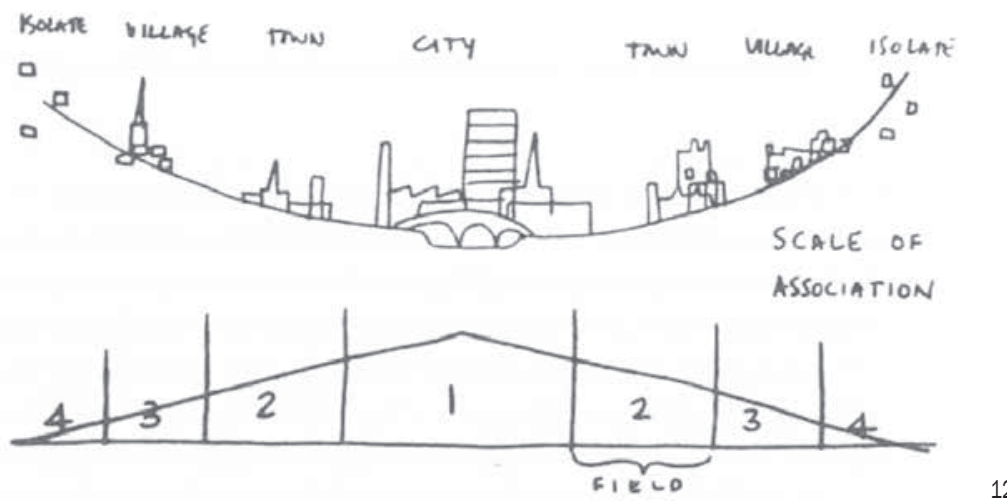

de Geddes" que constituyó el tema central del manifiesto

12. Sección valle del Manifiesto de Doorn. de Doorn'13 (figura 12). sto fue elaborado en 1954, tras el en- arquitectura vernácula las herramientas suficiegter sobre -Provence, en el contexto de las que crear el nuevo hábitat. Así afirmaban que "toda a situación nueva existe en el contexto de otras antiguas y

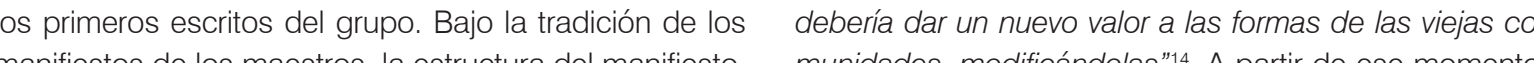

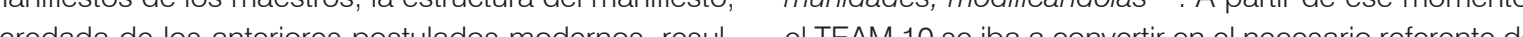

De esta forma y partiendo a modo de introducción de los planteamientos teóricos iniciales sobre la ciudad mo-

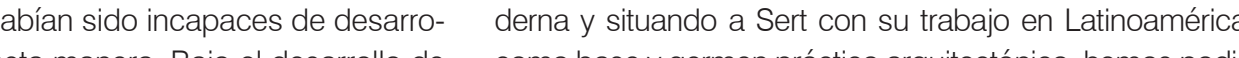

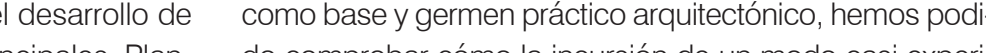
teaba que "para entender las pautas de las asociaciones menta humanas debemos considerar a cada comunidad en su en Marruecos o en barrios obreros de Londres dieronlugar entorno particular" No dejaba de ser un resumen de las a una nueva definición del concepto del hábitat contempofamosas rejillas expuestas el año anterior. Proponía un ráneo y moderno que los grandes maestros de la Historia análisis objetivo y permanente de la estructura humana de la Arcuitectura no habín sabido adantar completay de sus cambios Importante este punto asumiendo que mente a las nuevas condiciones tras la Segunda Guerra la arquitectura debía plantearse como ente adaptable y Mundial, hecho cronológico e histórico, que representó flexible ante las diversas situaciones que se planteen. La el verdadero punto de inflexión o cambio de rumbo en el estructura social a que se debía dar forma era mucho pensamiento arquitectónico tal y como aśl odescriben los más compleja que la conocida hasta entonces. El "viejo" diferentes textos criticos y teóricos sobre la materia n

13. En 1954, en la primera reunión del Team 10 se elabora el Manifiesto de Doorn. Se trata de uno de los primeros escritos del grupo.

1. Sólo tiene sentido considerar l a casa como parte de una comunidad, resultado de la interacción entre unos y otros.

2. No deberiamos perder el tiempo en catalogarar los elementos de la casa mientras
3. El "hábitat" se ocupa de la casa particular en un tipo de comunidad particular.
4. Las comunidades son las mismas en todas partes.

- La casa agrícola aislada

- Pueblo

- Ciudades pequeñas de varios tipos (nidst

-Grandes ciudades (multifincionales)

en la relación con su entorno (hábitat) en la sección del valle de Geddes.

ansporte del que se trate, su densidand cómoda -ha de tener facilidad de circulación-; consecuentemente, cualquiera que sea el tipo de

a sonte de que se trate, su densidad ha de crecer al ritmo de la población, por ejemplo: 1) tendrá menor densidad, 4) la mayor. ección valle.

8. Leadecuación de toda solución se ha de dar en el ámbito de la creación arquitectónica más bien que en el de la antropología social.

14. 8 .

\section{Bibliografía}

Capitel Antón: La arquitectura del patio. Barcelona: Gustavo Gili, 2005.

Candilis, Georges: "Habitat de type musulman à Casablanca" en L'Architecture d'Aujourd'hui. No60. 1955.

Capannini, Letizia: Habitat collectif méditerranéen et dynamique des espaces ouverts. Cas d'etude en Europe et en Afrique du Nord (1945-1970). En

Ecochard, Michel: "Urbanisme et construction pour le plus grand nombre”, En Architecture et Urbanisme, N06, 1950.

Ecochard, Michel: Casablanca ou Le Roman d'une ville. París: Editions de Pars, 1955.

Morales, José: La disolución de la estancia. Transformaciones domésticas 1930-1960. Madrid: Rueda, 2005.

Mumford, Eric: The CIAM discourse on urbanism, 1928-1960. Cambridge: MIT Press, 2000. PMCid:PMC1723562.

Mumford, Eric: Josep Luís Sert The architect of urban design, 1953-1969, Yale: Yale University Press, 2008.

Risselada, Max; Heuvel, Dirk van den: Team 10, 1953 - 1981 in search of utopia of the present. Rotterdam: NAi Publishers, 2005.

Sert, José Luis: Can our cities survive? : An ABC of urban problem, their analysis, their solutions; based on the proposalls formulated by the C.I.A.I.

Sert, José Luis: "Can patios make cities" En Architectural forum. No99, 1953

Smithson, Alison; Smithson, Peter: An Urban Project: “Golden Lane Housing. An Application of the Principles of Urban-re-identification”. En Architects

Smithson, Alison; Smithson, Peter: “The Built World: Urban Re-ldentification”. En Architectural Design. №6, 1955. pp.185-188.

Smithson, Alison; Smithson, Peter: "An Alternative to the Garden City Idea". En Architectural Design, No7, 1956.

Somer, Kees: The Functional city: the CIAM and Cornelis van Eesteren, 1928-1960. The Hague: EFL Foundation, 2007. PMCid:PMC3057183

Tournon Branly, Marion: "History of ATBAT and its influence on French Architecture". En Architectural Design, N¹, 1965. sidad Politechnica de Maand en 200. Becario FPu Universidad Politecrnica de Cartagena en 2008. Profesor Ayudante de Proyectos Arquitectonicicos en la Escuela de Arquitectura e Ingenierira de Edificación en la Universidad Politecticica de Cartagena en 2011. Realiza su tesis doctoral en el Departamento de Proyectos Arruitectonicicos de la Universidad Politécnica de Madrid. Pertenece al grupo de investigación "Habitar Colectivo" de la Universidad Politécnica de Cartagena. Ha colaborado con Amid/Cero9, Aranguren\&Gallegó Anutectos, Ezquiaga Arquitectura y Ecosistema Urbano entre otros.

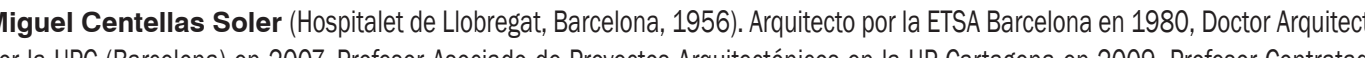
Doctor en 2010. Investigador principal del grupo de investigación "Habitar Colectivo" de la UPCT Su actividad profesional hatas sida publicada en las revistas ON, Geometría, Neutra y ARV y en Guías de Arquitectura de España. Director de Publicaciones del Colegio de Arquitectos de Almería: Documentos de Arquitectura, Archivos de Arquitectura y Deados. Miembro de la Comisión Técnica de la Fundación Docomomo lbérico.

Pedro García Martínez (Cartagena, Murcia, 1976). Arquitecto por la ETSA Madrid en 2004. Becario en el DPA de la ETSAM en 2007 (imparte clases en la Unidad Docente Mansilla//uñón y en el grupo Soto/Maroto). Becario de Investigación en la UPCT 2009. Profesor Ayudante en la UPCT en 2011. Su actividad profesional ha dado lugar a colaboraciones publicadas en El Croquis y Arquitec tura Via. Or sas en 


\section{Autor imagen y fuente bibliográfica de procedencia}

Información facilitada por los autores de los artículos: páginas 30 a 39, 1 a 7 (Restitución gráfica elaborada por Daniel Movilla Vega); página 41, 8 (Ginzburg, Moisei: Zhilishche: Opyt pyatiletney raboty nad problemoy zhilishcha. Moscú: Gosudarstvennoye nauchno tekhnicheskoye izd-vo stroitelnoy industrii i sudostroyeniya, 1934, p. 69), 9 (Sovremennaia Arkhitektura, SA. Moscú: nº 1, 1929, p. 13. Imágenes de planta: Ginzburg, Moisei: Zhilishche: Opyt pyatiletney raboty nad problemoy zhilishcha. Moscú: Gosudarstvennoye nauchno tekhnicheskoye izd-vo stroitelnoy industrii i sudostroyeniya, 1934, p. 73), 10 (Esquema de los tipos Sovremennaia Arkhitektura, SA. Moscú: $n^{0}$ 1, 1929, p. 2.Diagrama de eficacia Sovremennaia Arkhitektura, SA. Moscú: nº 1, 1929, p. 1); página 43, 11 y 12 (Restitución gráfica elaborada por Daniel Movilla Vega); página 44, 13 (V. Griuntal, en Narkomfin. Moscú: Museo de Arquitectura MUAR, 2008, p. 45), 14 ( Sovremennaia Arkhitektura, SA. Moscú: nº 5, 1929, p. 158); página 45 a 47, 15 a 18 (Restitución gráfica elaborada por Daniel Movilla Vega), página 47, 19 (Sovremennaia Arkhitektura, SA. Moscú: n 5, 1929, p. 17 ), 20 (Narkomfin. Moscú: Museo de Arquitectura MUAR, 2008, pp. 134, 69.); página 48 (Narkomfin. Moscú: Museo de Arquitectura MUAR, 2008, p. 135); páginas 52 a 55, 1 a 3 (Fernando Nieto Fernández); páginas 56 y 57, 4 a 6 (Kazuyo Sejima \& Ryue Nishizawa and Associates (SANAA). 1-5-27, Tatsumi, Koto-ku, Tokyo, 135-0053, Japón. Persona de contacto: Tomoko Fukuhara (press@sanaa.co.jp). Fecha de cesión y permiso: 02/08/2013); páginas 58 y 59, 7 a 9 (Chermayeff, Serge; Alexander, Christopher: Comunidad y privacidad. Hacia una nueva arquitectura humanista. Buenos Aires: Ediciones Nueva Visión, 1975); página 60, 10 (Lacaton \& Vassal Architectes. 206, Rue La Fayette, 75010, París, Francia (presse@lacatonvassal.com). Fecha de cesión y permiso: 01/07/2013); páginas 62 a 65, 11 a 14 (Fernando Nieto Fernández); página 66, 15 (Elemental S.A. Avda. Los Conquistadores, 1700, Piso 25, Providencia, Santiago, Chile. Persona de contacto: Rayna Razmilic (rr@elementalchile.cl). Fecha de cesión y permiso: 08/07/2013); página 69, 1 (Teiji Itoh Maisons anciennes au Japon), 2 http://muza-chan.net/aj/poze-weblog/shiodome-view-from-yurikamome-big.jpg. Foto bajo licencia Creative Commons); página 70, 3 (http://toky02050.com/en/ex1/01.html); páginas 72 a 76, 4 a 8 (Pedro Luis Gallego Fernández); página 77, 9 (http://www.designboom.com/architecture/ alx-junichi-sampei-on-the-cherry-blossom/); página 78, 10 (Pedro Luis Gallego Fernández); página 79, 11 (http://www.architectural-review.com/buildings/house-h-by-soufujimoto-architects-tokyo-japan/5218237.article); página 80, 12 (Pedro Luis Gallego Fernández), 13 (https://www.japlusu.com/shop/product/jutakutokushu-200909); página 81, 14 (Pedro Luis Gallego Fernández); página 82, 15 (http://www.mooponto.com/2013/06/29/yokohama-apartment-on-design-partners/); página 86, 1 (Mumford, Eric: Josep Lluís Sert The architect of urban design, 1953-1969, Yale: Yale University Press, 2008); página 88, 2 (Tournon Branly, Marion: “History of ATBAT and its influence on French Architecture". En Architectural Design, №1, 1965. p. 20), 3 (Ecochard, Michel: “Habitat musulman au Maroc”. En L 'Architecture d 'Aujourd 'hui, №60, 1955. p. 36); página 89, 4 (Ecochard, Michel: "Urbanisme et construction pour le plus grand nombre”. En Architecture et Urbanisme, №6, 1950. p. 9); página 90, 5 (www. isthaturban.wikispaces.com), 6 (Ecochard, Michel: “Habitat musulman au Maroc”. En L 'Architecture d 'Aujourd 'hui, №60, 1955. p. 38); página 91, 7 (www.isthaturban. wikispaces.com), 8 (Tournon Branly, Marion: History of ATBAT and its influence on French Architecture en Architectural Design, №1, 1965. p.21); página 92, 9 (Risselada, Max; Heuvel, Dirk van den: Team 10, 1953 - 1981 in search of utopia of the present. Rotterdam: NAi Publishers, 2005); 10 (Risselada, Max; Heuvel, Dirk van den: Team 10, 1953 - 1981 in search of utopia of the present. Rotterdam: NAi Publishers, 2005); página 93, 11 (Smithson, Alison; Smithson, Peter: "An Urban Project: Golden Lane Housing. An Application of the Principles of Urban-re-identification". En Architects Year Book, №5, 1953. p.50); página 94, 12 (Risselada, Max; Heuvel, Dirk van den: Team 10, 1953 - 1981 in search of utopia of the present. Rotterdam: NAi Publishers, 2005); página 97, 1 (@arquivo do Centro de Documentação 25 de Abril da Universidade de Coimbra. Colecção Direç̧ão Geral da Comunicação Social), 2 (@arquivo do Centro de Documentação 25 de Abril da Universidade de Coimbra. Colecção Alves Costa); página 101, 3 (@arquivo do Centro de Documentação 25 de Abril da Universidade de Coimbra. Colecção Alves Costa), 4 (Aitor Varea Oro. Oporto, marzo de 2011 -Antas-, septiembre de 2012 -Sé y São Victor); página 102, 5 (@arquivo do Centro de Documentação 25 de Abril da Universidade de Coimbra. Colecção Alves Costa), 6 (Fotografía de Manuel Días, facilitada por João Dias. Utilizada como imagen del documental "As Operações SAAL", de João Dias); página 104, 7 (Aitor Varea Oro. Redibujo de los planos originales consultados en el archivo profesional de Álvaro Siza Vieira); página 105, 8 (8: @BLOM Sistemas Geoespaciales, SLU), 9 y 10 (Boceto de Álvaro Siza. Archivo Álvaro Siza); página 108 y 109, 11 y 12 (Aitor Varea Oro. Redibujos de los planos originales consultados en el archivo profesional de Álvaro Siza Vieira); página 110, 13 (Fotógrafo sin identificar. Archivo Álvaro Siza); 14 (@arquivo do Centro de Documentação 25 de Abril da Universidade de Coimbra. Colecção Alves Costa); página 111, 15 (Aitor Varea Oro. Redibujo de los planos originales consultados en el archivo profesional de Álvaro Siza Vieira); página 112, 16 (Aitor Varea Oro. Oporto, marzo 2011); página 120, 1 (Copyright: Francis Frith Collection. Source: Lambeth Archives), 2 (Copyright: London Metropolitan Archives. Source: Lambeth Archives), 3 (Copyright and source: London Metropolitan Archives); página 121, 4 (Pablo Sendra, from the maps: County Series 1:2500 1854-1949. MAP EDITION: $1^{\text {st }}$ Revision 1893-1915. @ Crown Copyright and Landmark information Group Limited 2013. All rights reserved. 1886. Source: Digimap Historic), 5 (Pablo Sendra, from the maps: National grid 1:2500 1943-1995. MAP EDITION: National Survey 1943-1995 @ Crown Copyright and Landmark information Group Limited 2013. All rights reserved. 1952. Source: Digimap Historic); página 123, 6 (Pablo Sendra, from the maps: National grid 1:1250 1943-1993. MAP EDITION: 1st Revisions 1944-1993 @ Crown Copyright and Landmark information Group Limited 2013. All rights reserved. 1958-1974. Source: Digimap Historic), 7 (Pablo Sendra, from Ordnance Survey Maps ๑ Crown Copyright/database right 2013. An Ordnance Survey/EDINA supplied service); página 126, 8 (Pablo Sendra, from axial and segment maps provided by Space Syntax Ltd.);página 127, 9 (Pablo Sendra, from Ordnance Survey Maps @ Crown Copyright/database right 2013. An Ordnance Survey/EDINA supplied service); página 128, 10 (Copyright and source: London Metropolitan Archives), 11 a 14 (Pablo Sendra); páginas 133 a 148, 1 a 19 (María Prieto Peinado) 